\title{
The Physiological Response during Divergent Thinking
}

\author{
Gareth H. Loudon1,2*, Gina M. Deininger ${ }^{2}$ \\ ${ }^{1}$ Cardiff School of Art and Design, Cardiff Metropolitan University, Cardiff, UK \\ ${ }^{2}$ Centre for Creativity Ltd., Newport, UK \\ Email: *gloudon@cardiffmet.ac.uk
}

Received 16 November 2015; accepted 19 January 2016; published 22 January 2016

Copyright (C) 2016 by authors and Scientific Research Publishing Inc.

This work is licensed under the Creative Commons Attribution International License (CC BY). http://creativecommons.org/licenses/by/4.0/

\section{c) (i) Open Access}

\begin{abstract}
Our research studied the physiological response of participants during a creative task to investigate if a person's psychophysiological state was correlated with divergent thinking performance. We used heart rate variability as our physiological measure. We asked 50 participants to perform a cognitive task that assessed their divergent thinking skills and recorded their heart rate and heart rate variability (HRV) before and during the task. Frequency domain analysis was performed on the HRV. The results showed that there was a significant negative correlation between log-transformed low frequency HRV power and the number of "divergent thinking" words generated. Our results suggest that a person's psychophysiological state is correlated with their divergent thinking performance, and that attention and motivation may be important factors, however this needs further research. Our findings also suggest that being in a relaxed state before the start of a creative task is not a predictor of creative performance.
\end{abstract}

\section{Keywords}

Creativity, Divergent Thinking, Heart Rate Variability, Attention, Motivation

\section{Introduction}

Our broader research interests relate to the factors and processes affecting creativity including the psychophysiological state of a person [1]-[4]. In this study, we were interested in exploring whether there was a relationship between a person's physiological state and their divergent thinking performance. To do this, we asked participants to perform a cognitive task that assessed their divergent thinking skills and recorded their heart rate (HR) and heart rate variability (HRV) before and during the task.

\footnotetext{
${ }^{*}$ Corresponding author.
} 


\subsection{Creativity and Divergent Thinking}

One of the commonly agreed definitions of creativity is that "creativity is the ability to produce work that is both novel and appropriate" [5]. Another definition of creativity by Boden [6] is that "creativity is the ability to come up with ideas or artefacts that are new, surprising, and valuable" (p. 1). Guilford [7] proposes attributes of creativity that includes divergent thinking, where divergent thinking is defined as the exploration of many possible answers rather than thinking toward one right answer (convergent thinking). Kozbelt, Beghetto and Runco [8] say "divergent thinking occurs when ideas and associations move in varied directions, and as a result new and original ideas can be found. Convergent thinking, on the other hand, occurs when cognition is used to identify one correct or conventional answer” (p. 32). Divergent thinking relates to fluency (the quantity of ideas generated), flexibility (the variety of ideas), and originality (the uniqueness of ideas), but is not synonymous with creativity [9] as aspects of "value" are not considered. However, divergent thinking tests can be used as "estimates of the potential for creative thought" [9].

Runco and Chand [10] highlight that other contributing factors in creative thinking are knowledge and motivation and there has been some debate on the role of motivation, mood, and effort in relation to creativity [11]. A meta-analysis by Davis [11] on the relation between mood and creativity suggests that a positive mood enhances creativity. However, Silvia et al.'s [12] research into creative motivation, using cardiac autonomic markers of effort, suggests that people who show greater effort are significantly more creative while undertaking divergent thinking tasks.

Creativity is widely accepted as a cognitive event and cognition has predominantly been seen as being biologically grounded [13]. Our research interest is to investigate the relationship between a person's physiological state and their divergent thinking performance by analysing the number of "divergent thinking” words generated during a cognitive task. In addition, we are interested in exploring whether getting people into a relaxed state before the start of the creative task may have an effect on divergent thinking performance, and whether mental effort and attention during the creative task itself, estimated from heart rate variability measures, may be important.

\subsection{Heart Rate Variability}

Heart rate variability has been shown to be one of the most promising methods for measuring Autonomic Nervous System (ANS) activity [14] and is commonly regarded as a psychophysiological measure of mental and physical health [15] [16]. Heart rate variability (HRV) is the variation in the time interval between heartbeats (also known as the inter-beat intervals), and can be measured from the variation in consecutive R-R intervals of the Electrocardiogram (ECG) or be determined from consecutive pulse cycle intervals measured using techniques such as photoplethysmography [17]-[19].

Heart rate is regulated by the sympathetic and parasympathetic branches of the ANS. Sympathetic activity causes an increase in heart rate while parasympathetic activity causes a decrease in heart rate. Parasympathetic activity is mainly regulated by the vagus nerve and can result in relatively fast changes to the heart rate. Past research on the frequency analysis of heart rate variability has found that HRV frequencies in the high frequency (HF) range $(0.15-0.4 \mathrm{~Hz})$ relate to the variation in the heart rate due to the breathing cycle, also known as respiratory sinus arrhythmia (RSA), and HRV frequencies in the low frequency (LF) range $(0.04-0.15 \mathrm{~Hz})$ relate to the regulation of blood pressure [20]-[22]. Variability in heart rate below $0.04 \mathrm{~Hz}$, normally labelled as very low frequency (VLF), could relate to a range of factors, and although these very low frequencies might well have important psychophysiological information, their interpretation still remains unclear [20] [16].

High frequency (HF) HRV is usually associated with purely parasympathetic activity. However low frequency (LF) HRV is thought to be influenced by both sympathetic and parasympathetic activity [23]-[25], with recent studies suggesting that LF HRV power relates more to baroreflex outflow [26] [27] and vagal control [22].

Several researchers including Friedman [28] and Murakami and Ohira [29] have showed that HF HRV power reduces, heart rate increases and LF HRV power increases when people are under stress or are suffering from anxiety, indicating reduced parasympathetic activity.

Past research studies on mental workload and attention have shown that an increase in mental workload and attention causes a reduction in HRV and an increase in heart rate [30]-[32]. In addition, engagement with cognitive, working memory tasks reduces power in the LF range of the HRV [31] [33] [34]. Studies by Van Roon et 
al. [32] also show a reduction in low and high frequency HRV under mental workload and suggest that this is due to a reduction in parasympathetic activity and an increase in sympathetic activity.

Capa et al. [35] investigated the relationship between motivation and task difficulty on mental effort and found that participants who had high motivation to achieve success (approach-driven), compared to those who had high motivation to avoid failure (avoidance-driven), performed better and had a larger reduction in their LF HRV, especially during a difficult task. Silvia et al. [12], in their study on creative motivation, found that people with higher creative achievement generated more creative ideas and showed higher levels of effort. In addition, their study found tentative support for a link between sympathetic activity and divergent thinking performance.

\subsection{Research Study Aims and Objectives}

Our main aim in this research study was to record and analyse the physiological response of participants during a divergent thinking task to:

1) Investigate if a person's psychophysiological state is correlated with divergent thinking performance.

2) Explore whether getting people into a relaxed state before the start of the creative task might have an effect on divergent thinking performance.

\section{Methods}

\subsection{Participants}

A total of 50 participants took part in the study, 35 males (70\%) and 15 females (30\%). Ethnic representation in the sample was $90 \%$ White, $6 \%$ Asian, $2 \%$ Black and $2 \%$ Eurasian. Criteria for inclusion in the study were that participants were aged between 18 and 22 years of age, and were students at the Cardiff School of Art and Design. Ethics approval for the study was given by the Cardiff School of Art and Design's Research Ethics Committee. All participants gave their informed consent prior to their inclusion in the study.

\subsection{Procedure}

We used the same creative task as the one used by Dijksterhuis and Meurs [36] in Experiment 1 of their study into creativity and unconscious thought. As described in their experiment, participants were instructed to generate new names for pasta. Each participant undertook the creative task in a quiet separate room and instructions for the exercise were given in printed format. The study was conducted during the day and all participants were seated during the task to reduce the effect of variation in respiration rates [37]. Pulse cycle interval data of each participant was recorded using the emWave 2 sensor by Heart Math [38]. The sensor was clipped to the participant's ear and the recording system checked for accuracy before the start of any tasks.

Male and female participants were randomly allocated to one of two conditions. 7 females and 18 males were assigned to the first condition and 8 females and 17 males were assigned to the second condition. This was done to reduce the effect of variation in the distribution of males and females between the two conditions. In the first condition (immediate), participants were given the instructions related to the task and given one minute to come up with as many new pasta names as they could think of, which they had to write down on a sheet of paper. On the printed instructions, five examples were given of existing pasta names and all examples ended with the letter "i". The idea being that the priming of the participants with pasta names that all end in the letter "i" might influence them to come up with "conventional" answers that also end in the letter "i". Any new pasta names ending in the letter "i" would be categorised as "convergent thinking" names. More "varied" pasta names not ending in the letter "i" would be categorised as "divergent thinking" names.

In the second condition (relaxed), participants were initially asked to relax and focus on breathing slowly. Their heart rate variability was monitored in real-time using the emWave Pro software [39]. The idea was that as the participants relaxed and focused on breathing slowly their RSA would shift towards the LF range and we could use this measure as a guide to their level of relaxation and slow breathing. When the shift in power to the LF HRV went above a set threshold defined in the emWave Pro software, participants were classed as being in a "relaxed" state. Once in this "relaxed" state, participants were given the instructions related to the task and given one minute to come up with as many new pasta names as they could think of, which they had to write down on a sheet of paper (i.e. the exact same instructions as participants in the first condition).

The reason for separating the participants into two conditions was to gain answers related to objective two- 
whether getting people into a relaxed state before the start of the creative task might have an effect on divergent thinking performance.

\subsection{Data Analysis and HRV Measures}

For each of the 50 participants, the number of pasta names categorised as divergent thinking (Td) and convergent thinking (Tc) names were recorded.

All the pulse cycle interval data recorded for each of the 50 participants was exported from the emWave Pro software into the HRV analysis software, KUBIOS version 2.2 [40]. After visual inspection of the data, artefact correction was performed on 5 of the 50 pulse cycle interval samples using the KUBIOS software to correct intervals differing by more than 0.35 seconds from the local mean interval using a piecewise cublic spline interpolation method. The mean heart rate over the one-minute task was recorded for each participant. We chose to use frequency domain analysis of the HRV as it is a commonly used method for studying sympathetic and parasympathetic activity [20] [14] [16] and it also allowed us to compare our results against the results of other key research more easily.

The KUBIOS software converted the pulse cycle interval data into equidistant samples using an interpolation method and then calculated the power spectrum density (PSD) estimates of HRV using Welch's Fast Fourier Transform (FFT) method. PSD estimates were recorded for both LF ranges and HF ranges. In our study we set the HF range to $0.15-0.4 \mathrm{~Hz}$ and the LF range to $0.07-0.15 \mathrm{~Hz}$ rather than the more common LF range of 0.04 - $0.15 \mathrm{~Hz}$. This was because our creative task only lasted one minute, and frequencies below $0.07 \mathrm{~Hz}$ would have had a limited number of samples to form reliable spectral information. We didn't want to have our creative task longer than one minute as we felt that this might reduce the effect of the relaxed condition compared to the immediate condition and put more emphasize on sustained attention and effort during the task.

Both the PSD estimates for the LF and HF ranges were natural-log transformed to obtain Gaussian distributions [41]. Statistical analysis was conducted using SPSS Version 22. The Pearson product-moment correlation coefficient was used to study the relationships among the variables under study to gain an answer to objective one, i.e. whether there was a correlation between divergent thinking performance and physiological measures recorded using HRV. In addition, an analysis of variance (ANOVA) was conducted to study the difference between means from a number of variables in relation to different factors. These included ANOVAs investigating the difference in means between the physiological measures recorded for the participants in the relaxed condition during the relaxation period and during the task; investigating the difference in means between the number of "divergent thinking" words for the two conditions (relaxed and immediate) to gain an answer to objective two, i.e. whether getting people into a relaxed state before the start of the creative task might have an effect on divergent thinking performance; and ANOVAs studying the differences in the LF and HF measures for the two conditions.

The LF and HF measures were also analysed using an ANOVA based on the median split of the number of "divergent thinking" words generated for all 50 participants. This resulted in LF and HF measures for two groups; group one for the 25 participants producing the lowest number of "divergent thinking” words (Low Td) and group two for the 25 participants producing the highest number of "divergent thinking” words (High Td). This was undertaken to investigate further how the divergent thinking performance might relate to the physiological measures recorded.

The alpha level was set at 0.05 for the classification of significant difference and at 0.10 for marginal significance. Based on Cohen [42] we interpreted effect size for correlations as small when $r=0.1$; medium when $r=$ 0.3 ; and large when $r=0.5$. For ANOVA we interpreted effect size as small when Cohen's $d=0.2$; medium when $d=0.5$; and large when $d=0.8$.

\section{Results}

\subsection{Effects of Initial Relaxation on HRV}

Table 1 below shows the physiological measures for the 25 participants who were in the relaxed condition while they were "relaxing" before the start of the task, and during the task. On average, participants spent 2 minutes 56 seconds "relaxing" before the start of the task. There was variation in the time given to participants to relax, as some participants needed a longer time to get into a "relaxed" state (as defined by our measure). The values 
Table 1. Physiological measures for the relaxed condition before and during the task.

\begin{tabular}{|c|c|c|c|c|c|c|c|}
\hline \multirow{2}{*}{ Measure } & \multicolumn{2}{|c|}{ During Relaxation } & \multicolumn{2}{|c|}{ During Task } & \multirow[b]{2}{*}{$\mathrm{F}$} & \multirow[b]{2}{*}{$p$} & \multirow[b]{2}{*}{ Effect Size } \\
\hline & Mean & SD & Mean & SD & & & \\
\hline HR & 76.24 & 11.07 & 83.68 & 11.57 & 26.91 & $<0.01$ & 0.66 \\
\hline $\ln \mathrm{LF}$ & 7.20 & 1.32 & 6.58 & 0.99 & 3.31 & 0.08 & 0.53 \\
\hline $\ln \mathrm{HF}$ & 7.62 & 0.98 & 6.83 & 1.02 & 16.21 & $<0.01$ & 0.79 \\
\hline
\end{tabular}

HR is Heart Rate, ln LF is log transformed absolute low frequency power, ln HF is log transformed absolute high frequency power. Note: Effect size is measured using Cohen's d.

shown in Table 1, under the heading "During Relaxation", are calculated based on the last minute spent in the "relaxed" state before the start of the task.

There was a significant difference between the heart rate of the participants during the last minute of relaxation and during the task $[\mathrm{F}(1,48)=26.91, p<0.01]$ with a medium effect size, with the mean heart rate increasing during the task; there was a marginally significant difference between the log transformed absolute LF power (ln LF) for the two conditions $[F(1,48)=3.31, p=0.08]$ with a medium effect size, with the mean $\ln$ LF reducing during the task; and there was a significant difference between the log transformed absolute HF power (ln HF) for the two conditions [F $(1,48)=16.21, p<0.01]$ with a medium effect, with the mean ln HF reducing during the task.

When analysing the changes in ln LF and ln HF it is important to ask whether respiration can account for some of these effects [38]. As recommended by Allen et al. [38], respiration was estimated from the peak of the spectral information of the inter-beat intervals, with the average respiration rate being $0.15 \mathrm{~Hz}$ during relaxation and $0.19 \mathrm{~Hz}$ during the task. This is a significant difference $[\mathrm{F}(1,48)=4.13, p=0.05]$ with a medium effect size.

\subsection{Effects of Initial Relaxation on Divergent Thinking}

Table 2 below shows the results of the number of "divergent thinking” words, "convergent thinking” words and total words generated (Td, Tc and Tw respectively) for the two conditions (immediate and relaxed) along with different physiological measures including heart rate during the task (HR), log transformed absolute LF power (ln LF), and log transformed absolute HF power (ln HF).

There was no significant difference between the number of "divergent thinking" words (Td), "convergent thinking" words (Tc) or total number of words (Tw) generated for the two conditions. In addition, no other significant effects of condition were present for the other measures. Again, respiration was estimated from the peak of the spectral information of the inter-beat intervals, with the average respiration rate being $0.23 \mathrm{~Hz}$ for the immediate condition and $0.19 \mathrm{~Hz}$ for the relaxed condition. This is a marginally significant difference $[\mathrm{F}(1,48)$ $=3.52, p=0.07]$ with a medium effect size.

\subsection{Effects of Heart Rate on Divergent Thinking}

When comparing the heart rate of participants during the one-minute task (HR) against the number of "divergent thinking” words (Td) they generated, for all 50 participants, there was no significant correlation. Pearson's correlation, $r=0.02, p$ (2-tailed) $=0.91, \mathrm{~N}=50$, representing a small effect size.

\subsection{Effects of HRV on Divergent Thinking}

When comparing the log transformed absolute LF power (ln LF) of participants during the one-minute task against the number of "divergent thinking” words (Td) they generated, for all 50 participants, there was a significant negative correlation. Pearson's correlation, $r=-0.47, p$ (2-tailed) $<0.01, \mathrm{~N}=50$, representing a large effect size. When comparing the log transformed absolute HF power (ln HF) of participants during the oneminute task against the number of "divergent thinking" words (Td) they generated, for all 50 participants, there was a marginal, negative correlation. Pearson's correlation, $r=-0.26, p$ (2-tailed) $=0.06, \mathrm{~N}=50$, representing a medium effect size. There was no significant correlation between the total number of words generated (Tw) and 
Table 2. Word numbers and physiological measures for the two conditions.

\begin{tabular}{ccccccccc}
\hline \multirow{2}{*}{ Measure } & \multicolumn{2}{c}{ Immediate Condition } & \multicolumn{2}{c}{ Relaxed Condition } & & & \\
\cline { 2 - 8 } & Mean & SD & Mean & SD & F & $p$ & Effect Size \\
\hline Td & 1.84 & 2.03 & 1.92 & 1.44 & 0.03 & 0.87 & 0.05 \\
Tc & 3.64 & 2.08 & 3.52 & 2.63 & 0.03 & 0.86 & 0.05 \\
Tw & 5.48 & 2.02 & 5.44 & 2.38 & $<0.01$ & 0.95 & 0.02 \\
HR & 78.84 & 11.61 & 83.68 & 11.57 & 2.18 & 0.15 & 0.43 \\
ln LF & 6.53 & 1.09 & 6.58 & 0.99 & 0.04 & 0.85 & 0.06 \\
In HF & 6.74 & 1.07 & 6.83 & 1.02 & 0.10 & 0.75 & 0.09 \\
\hline
\end{tabular}

Td is number of "divergent thinking” words generated, Tc is number of "convergent thinking” words generated, Tw is total number of words generated, HR is Heart Rate, ln LF is log transformed absolute low frequency power, and ln HF is log transformed absolute high frequency power. Note: Effect size is measured using Cohen’s d.

$\ln \mathrm{LF}(r=-0.12, p$ (2-tailed) $=0.39, \mathrm{~N}=50)$ and between Tw and $\ln \mathrm{HF}(r=0.18, p(2$-tailed $)=0.21, \mathrm{~N}=50)$. There was a significant, positive correlation between $\ln$ LF and $\ln$ HF. Pearson's correlation, $r=0.57, p$ (2-tailed) $<0.01, N=50$, representing a large effect size.

Table 3 below compares the results of the number of "divergent thinking" words (Td), "convergent thinking" words (Tc) and total number of words (Tw) generated along with log transformed absolute LF power (ln LF), and log transformed absolute HF power (ln HF) for two groups based on the median split of the number of "divergent thinking" words generated for all 50 participants. Group one includes the 25 participants producing the lowest number of "divergent thinking" words (Low Td); and group two includes the 25 participants producing the highest number of "divergent thinking" words (High Td).

Unsurprisingly, there was a significant difference between the numbers of "divergent thinking" words (Td) generated between the two groups $[F(1,48)=78.15, p<0.01]$, with a large effect size. There was no significant difference between the total numbers of words (Tw) generated between the two groups, however there was a significant difference between the numbers of "convergent thinking" words $(\mathrm{Tc})$ generated $[\mathrm{F}(1,48)=15.20, p$ $<0.01]$, with a large effect size. For $\ln$ LF there was a significant difference between the two groups $[\mathrm{F}(1,48)=$ $13.31, p<0.01]$ with a large effect size, with the average ln LF being significantly lower in the group with the highest number of "divergent thinking" words (High Td), (Figure 1). The average ln HF was also lower in the group with the highest number of "divergent thinking" words (High Td) but this did not reach significance (Figure 2), but did have a medium effect size. The average respiration rate (as estimated from the peak of the spectral information of the inter-beat intervals) was $0.21 \mathrm{~Hz}$ for the Low Td group and $0.22 \mathrm{~Hz}$ for the High Td group. There was no significant difference between the two groups $[F(1,48)=0.07, p=0.80]$.

\section{Discussion}

\subsection{Effects of Initial Relaxation on HRV}

The results suggest that the 25 participants in the "relaxed" condition were more relaxed during the relaxation period compared to the time they were undertaking the task with, on average, a lower heart rate and higher HF power (ln HF), suggesting higher parasympathetic activity. Estimates of the respiration rate also suggest that there were slower respiration rates, on average, during the relaxation period compared to the time during the task and this might partially account for the higher LF power (ln LF) during the relaxation period. The increase in heart rate during the task is in line with the findings from other studies associated with mental workload and suggests an increase in sympathetic activity and increased arousal [30] [32] [40].

\subsection{Effects of Initial Relaxation on Divergent Thinking}

The physiological measures recorded during the task, including heart rate (HR), LF power (ln LF) and HF power (ln HF) suggest that, during the task itself, there was no significant difference between the two conditions (relaxed and immediate). Therefore, a participant being relaxed before the start of the task was not a predictor of their physiological state during the task. In addition, there was no significant difference between the numbers of 


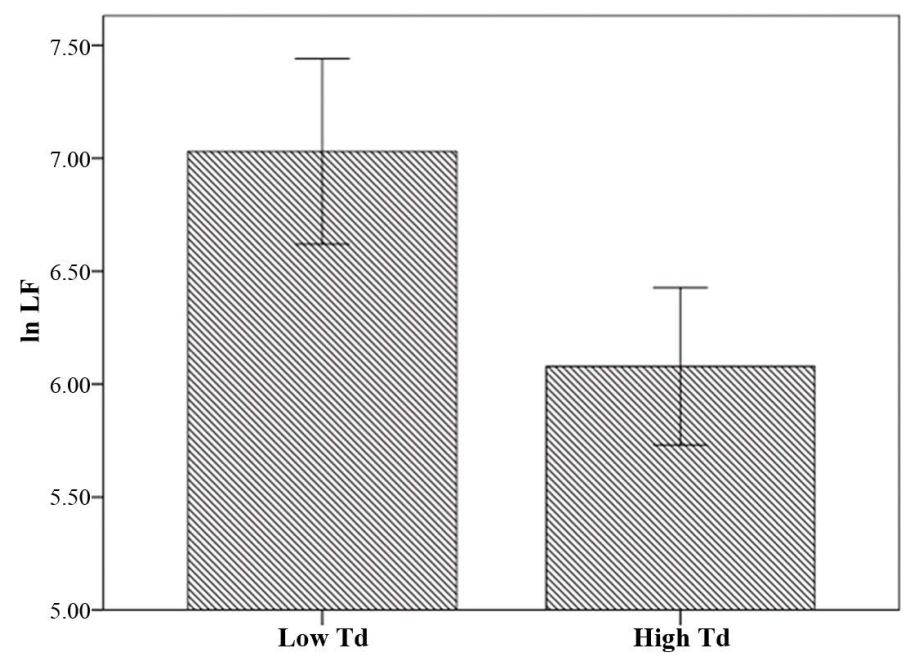

Figure 1. The mean log transformed absolute low frequency power (ln LF) for the two groups, low (Low Td) and high (High Td) numbers of “divergent thinking” words. Error bars: 95\% Confidence Intervals.

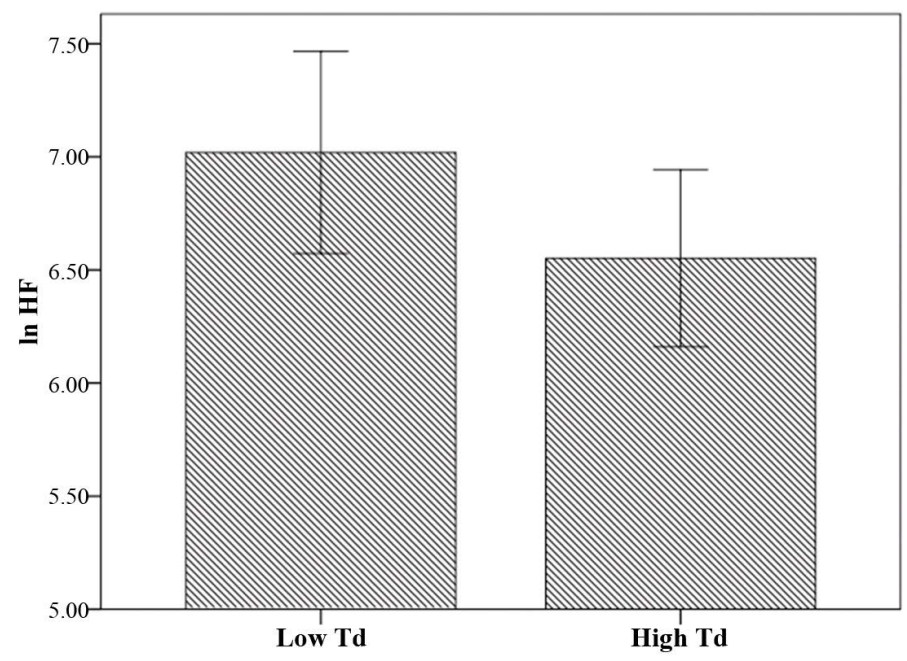

Figure 2. The mean log transformed absolute high frequency power (ln HF) for the two groups, low (Low Td) and high (High Td) numbers of “divergent thinking” words. Error bars: 95\% Confidence Intervals.

Table 3. Comparison of physiological measures between groups with a low and high number of divergent thinking words.

\begin{tabular}{|c|c|c|c|c|c|c|c|}
\hline \multirow{2}{*}{ Measure } & \multicolumn{2}{|c|}{ Low Td } & \multicolumn{2}{|c|}{ High Td } & \multirow[b]{2}{*}{ F } & \multirow[b]{2}{*}{$p$} & \multirow[b]{2}{*}{ Effect Size } \\
\hline & Mean & SD & Mean & SD & & & \\
\hline $\mathrm{Td}$ & 0.52 & 0.65 & 3.24 & 1.39 & 78.15 & $<0.01$ & 2.55 \\
\hline Tc & 4.72 & 2.22 & 2.44 & 1.89 & 15.20 & $<0.01$ & 1.11 \\
\hline Tw & 5.24 & 2.13 & 5.68 & 2.27 & 0.50 & 0.48 & 0.20 \\
\hline $\ln L F$ & 7.03 & 1.00 & 6.08 & 0.84 & 13.31 & $<0.01$ & 1.05 \\
\hline $\ln \mathrm{HF}$ & 7.02 & 1.08 & 6.55 & 0.95 & 2.65 & 0.11 & 0.47 \\
\hline
\end{tabular}

Td is number of "divergent thinking” words generated, Tc is number of "convergent thinking” words generated, Tw is total number of words generated, $\ln$ LF is log transformed absolute low frequency power, and ln HF is log transformed absolute high frequency power. Note: Effect size is measured using Cohen's d. 
“divergent thinking” words (Td) generated for the two conditions, which maybe not that surprising considering the similarity of physiological measures between the two conditions during the task. Therefore, getting people into a relaxed state before the start of a creative task does not have an effect on their divergent thinking performance. Our previous research on the effect of play on creative problem solving [3] suggested that being in a playful state before undertaking the task helped in performance. Other research has also found a link between positive mood and creative performance [11]. However, the difference in findings could be that a playful state is more about positive valence and high arousal rather than relaxation. This needs further investigation.

\subsection{Effects of Heart Rate on Divergent Thinking}

There was no significant correlation between the heart rate during the task (HR) and the number of "divergent thinking” words (Td), suggesting that this is not a determining factor for divergent thinking performance. However further research is required to study within-person effects from baseline to task to gain a deeper understanding of the importance of the change in heart rate.

\subsection{Effects of HRV on Divergent Thinking}

The significant negative correlation between LF power (ln LF) and the number of "divergent thinking" words (Td) suggests that divergent thinking performance might be related to the level of mental workload and attention applied to the task, as similar reductions in LF power have been shown in other mental workload tasks [31]-[33] [43]. The marginal, negative correlation between HF power (ln HF) and the number of "divergent thinking" words (Td) might also be caused by the same effect of mental workload and attention as shown in previous studies [32] [44]. Our findings might also relate to the level of motivation shown by the participants, as suggested by Silvia et al. [12] and Capa et al. [35]. It is important to note, however, that there was not a significant correlation between the total number of words generated and either LF power (ln LF) or HF power (ln HF). Therefore, the results seem to suggest that performance is not just about mental effort. This needs further investigation.

\section{Conclusion}

Our results suggest that a person's psychophysiological state is correlated with their divergent thinking performance. However, the interpretation of the findings needs further research, for example, the importance of motivation, valence, mental effort and attention in creativity and how these different factors may interact.

\section{Limitations and Future Directions}

One of the limitations of the study was that each task only lasted one minute, and therefore only one minute of HRV data was analysed to cover the task duration. The recommendation by the Task Force of the European Society of Cardiology and the North American Society of Pacing and Electrophysiology [21] is that at least two minutes of data be used to analyse both LF and HF HRV so that all the spectral information is reliable. Signals at $0.04 \mathrm{~Hz}$ would only be seen 2.4 times in one minute. This was mitigated to some extent by limiting the LF range to $0.07-0.15 \mathrm{~Hz}$ rather than the more common $0.04-0.15 \mathrm{~Hz}$ range.

A relatively small sample size of 50 participants in the study means that any conclusions drawn from the results should be tentative. Further studies are required by other researchers to see if the results found in this study are replicated.

We plan to conduct further studies on the relation between attention, play and a person's psychophysiological state to help answer some of the questions raised from this research study and our previous research studies on play [1] [3]. We also plan to conduct further studies on the relation between "flow" [45], creativity and a person’s psychophysiological state.

\section{References}

[1] Deininger, G.M., Loudon, G.H. and Norman, S. (2012) Modal Preferences in Creative Problem Solving. Cognitive Processing, 13, 147-150. http://dx.doi.org/10.1007/s10339-012-0479-3

[2] Deininger, G.M. (2013) Does State of Being and Dynamic Movement Have a Relationship with Creativity? PhD Thesis, Cardiff Metropolitan University, Cardiff. 
[3] Loudon, G.H., Deininger, G.M. and Gordon, B.S. (2012) Play, Autonomy and the Creative Process. In: Duffy, A., Nagai, Y. and Taura, T., Eds., Proceedings of the 2nd International Conference on Design Creativity, The Design Society, 87-96.

[4] Loudon, G.H. and Deininger, G.M. (2014) A New Model for Supporting Creativity in Research Organisations. In: Schimpf, S., Ed., Proceedings of the R\&D Management Conference, Fraunhofer IAO, Stuttgart, 93-100.

[5] Sternberg, R.J. and Lubart, T.I. (1999) The Concept of Creativity: Prospects and Paradigms. In: Sternberg, Ed., Handbook of Creativity, Cambridge University Press, Cambridge, 169-186.

[6] Boden, M.A. (2004) The Creative Mind: Myths and Mechanisms. 2nd Edition, Routledge, London, 1.

[7] Guilford, J.P. (1950) Creativity. American Psychologist, 5, 444-454. http://dx.doi.org/10.1037/h0063487

[8] Kozbelt, A., Beghetto, R.A. and Runco, M.A. (2010) Theories of Creativity. In: Kaufman, J.C. and Sternberg, R.J., Eds., Cambridge Handbook of Creativity, Cambridge University Press, New York, 20-47. http://dx.doi.org/10.1017/cbo9780511763205.004

[9] Runco, M.A. (2008) Commentary: Divergent Thinking Is Not Synonymous with Creativity. Psychology of Aesthetics, Creativity and the Arts, 2, 93-96. http://dx.doi.org/10.1037/1931-3896.2.2.93

[10] Runco, M.A. and Chand, I. (1995) Cognition and Creativity. Educational Psychology Review, 7, 243-267. http://dx.doi.org/10.1007/BF02213373

[11] Davis, M.A. (2009) Understanding the Relationship between Mood and Creativity: A Meta-Analysis. Organizational Behavior and Human Decision Processes, 108, 25-38. http://dx.doi.org/10.1016/j.obhdp.2008.04.001

[12] Silvia, P.J., Beaty, R.E., Nusbaum, E.C., Eddington, K.M. and Kwapil, T.R. (2014) Creative Motivation: Creative Achievement Predicts Cardiac Autonomic Markers of Effort during Divergent Thinking. Biological Psychology, 102, 30-37. http://dx.doi.org/10.1016/j.biopsycho.2014.07.010

[13] Kaufman, A.B., Kornilov, S.A., Bristol, A.S., Tan, M. and Grigorenko, E.L. (2010) The Neurobiological Foundation of Creative Cognition. In: Kaufman, J.C. and Sternberg, R.J., Eds., Cambridge Handbook of Creativity, Cambridge University Press, Cambridge, 216-232. http://dx.doi.org/10.1017/cbo9780511763205.014

[14] Malik, M. (1996) Heart Rate Variability: Standards of Measurement, Physiological Interpretation, and Clinical Use: Task Force of the European Society of Cardiology and the North American Society for Pacing and Electrophysiology. Annals of Noninvasive Electrocardiology, 1, 151-181. http://dx.doi.org/10.1111/j.1542-474X.1996.tb00275.x

[15] Kemp, A.H. and Quintana, D.S. (2013) The Relationship between Mental and Physical Health: Insights from the Study of Heart Rate Variability. Int. J. Psychophysiology, 89, 296-304. http://dx.doi.org/10.1016/j.ijpsycho.2013.06.018

[16] Xhyheri, B., Manfrini, O., Mazzolini, M., Pizzi, C. and Bugiardini, R. (2012) Heart Rate Variability Today. Progress in Cardiovascular Diseases, 55, 321-331. http://dx.doi.org/10.1016/j.pcad.2012.09.001

[17] Schäfer, A. and Jan Vagedes, J. (2013) How Accurate Is Pulse Rate Variability as an Estimate of Heart Rate Variability? A Review on Studies Comparing Photoplethysmographic Technology with an Electrocardiogram. International Journal of Cardiology, 166, 15-29. http://dx.doi.org/10.1016/j.ijcard.2012.03.119

[18] Gil, E., Orini, M., Bailón, R., Vergara, J.M., Mainardi, L. and Laguna, P. (2010) Photoplethysmography Pulse Rate Variability as a Surrogate Measurement of Heart Rate Variability during Non-Stationary Conditions. Physiological Measurement, 31, 1271-1290. http://dx.doi.org/10.1088/0967-3334/31/9/015

[19] Lu, G., Yang, F., Taylor, J.A. and Stein, J.F. (2009) A Comparison of Photoplethysmography and ECG Recording to Analyse Heart Rate Variability in Healthy Subjects. Journal of Medical Engineering \& Technology, 33, 634-641. http://dx.doi.org/10.3109/03091900903150998

[20] Berntson, G., Bigger Jr., J., Eckberg, D., Grossman, P., Kaufmann, P., Malik, M., Nagaraja, H., Porges, S., Saul, J., Stone, P. and van der Molen, M. (1997) Heart Rate Variability: Origins, Methods, and Interpretive Caveats. Psychophysiology, 34, 623-648. http://dx.doi.org/10.1111/j.1469-8986.1997.tb02140.x

[21] Task Force of the European Society of Cardiology and the North American Society of Pacing and Electrophysiology (1996) Head Rate Variability: Standards of Measurement, Physiological Interpretation, and Clinical Use. Circulation, 93, 1043-1065. http://dx.doi.org/10.1161/01.CIR.93.5.1043

[22] Reyes Del Paso, G.A., Langewitz, W., Mulder, L.J.M., Van Roon, A. and Duschek, S. (2013) The Utility of Low Frequency Heart Rate Variability as an Index of Sympathetic Cardiac Tone: A Review with Emphasis on a Reanalysis of Previous Studies. Psychophysiology, 50, 477-487. http://dx.doi.org/10.1111/psyp.12027

[23] Akselrod, S., Gordon, D., Madwed, J.B., Snidman, N.C., Shannon, D.C. and Cohen, R.J. (1985) Hemodynamic Regulation: Investigation by Spectral Analysis. American Journal of Physiology, 18, 867-875.

[24] Koh, J., Brown, T.E., Beightol, I.A., Ha, C.Y. and Eckberg. D.L. (1994) Human Autonomic Rhythms: Vagal Cardiac Mechanisms in Tetraplegia Subjects. Journal of Physiology, 474, 481-495. http://dx.doi.org/10.1113/jphysiol.1994.sp020039 
[25] Pomeranz, B., Macaulay, R.J., Caudill, M.A., Kutz, I., Adam, D., Gordon, D., Kilborn, K.M., Barger, A.C., Shannon, D.C., Cohen, R.J. and Benson, H. (1985) Assessment of Autonomic Function in Humans by Heart Rate Spectral Analysis. American Journal of Physiology, 248, 151-153.

[26] Goldstein, D., Bentho, O., Park, M. and Sharabi, Y. (2011) Low-Frequency Power of Heart Rate Variability Is Not a Measure of Cardiac Sympathetic Tone but May Be a Measure of Modulation of Cardiac Autonomic Outflows by Baroreflexes. Experimental Physiology, 96, 1255-1261. http://dx.doi.org/10.1113/expphysiol.2010.056259

[27] Moak, J., Goldstein, D., Eldadah, B., Saleem, A., Holmes, C., Pechnik, S. and Sharabi, Y. (2009) Supine Low-Frequency Power of Heart Rate Variability Reflects Baroreflex Function, Not Cardiac Sympathetic Innervations. Cleveland Clinic Journal of Medicine, 76, 51-59. http://dx.doi.org/10.3949/ccjm.76.s2.11

[28] Friedman, B.H. (2007) An Autonomic Flexibility-Neurovisceral Integration Model of Anxiety and Cardiac Vagal Tone. Biological Psychology, 74, 185-199. http://dx.doi.org/10.1016/j.biopsycho.2005.08.009

[29] Murakami, H. and Ohira, H. (2007) Influence of Attention Manipulation on Emotion and Autonomic Responses. Perceptual and Motor Skills, 105, 299-308. http://dx.doi.org/10.2466/pms.105.5.299-308

[30] Althaus, M., Mulder, L.J.M., Mulder, G., Van Roon, A.M. and Minder, R.B. (1998) Influence of Respiratory Activity on the Cardiac Response Pattern to Mental Effort. Psychophysiology, 35, 420-430. http://dx.doi.org/10.1111/1469-8986.3540420

[31] Tripathi, K.K., Mukundan, C.R. and Lazar Mathew, T. (2003) Attentional Modulation of Heart Rate Variability (HRV) during Execution of PC Based Cognitive Tasks. Indian Journal of Aerospace Medicine, 47, 1-10.

[32] Van Roon, A.M., Mulder, L.J.M., Althaus, M. and Mulder, G. (2004) Introducing a Baroreflex Model for Studying Cardiovascular Effects of Mental Workload. Psychophysiology, 41, 961-981. http://dx.doi.org/10.1111/j.1469-8986.2004.00251.x

[33] Boucsein, W. and Backs, R.W. (2000) Engineering Psychophysiology as a Discipline: Historical and Theoretical Aspects. In: Backs, R.W. and Boucsein, W., Eds., Engineering Psychophysiology: Issues and Applications, Lawrence Erlbaum Associates, London, 3-30.

[34] Veltman, J.A. and Gaillard, A.W.K. (1996) Physiological Indices of Workload in a Simulated Flight Task. Biological Psychology, 42, 323-342. http://dx.doi.org/10.1016/0301-0511(95)05165-1

[35] Capa, R.L., Audiffren, M.A. and Ragot, S. (2008) The Interactive Effect of Achievement Motivation and Task Difficulty on Mental Effort. International Journal of Psychophysiology, 70, 144-150. http://dx.doi.org/10.1016/j.ijpsycho.2008.06.007

[36] Dijksterhuis, A. and Meurs, T. (2006) Where Creativity Resides: The Generative Power of Unconscious Thought. Consciousness and Cognition, 15, 135-146. http://dx.doi.org/10.1016/j.concog.2005.04.007

[37] Allen, J.J.B., Chambers, A.S. and Towers, D.N. (2007) The Many Metrics of Cardiac Chronotropy: A Pragmatic Primer and a Brief Comparison of Metrics. Biological Psychology, 74, 243-262. http://dx.doi.org/10.1016/j.biopsycho.2006.08.005

[38] emWave2 (2015) Heart Rate Monitor. http://www.heartmathstore.com/category/emWave2/

[39] emWave Pro (2015) Version 3.3, Heart Rate Variability Software. http://store.heartmath.com/emwavepro

[40] Tarvainen, M. and Niskanen, J. (2008) Kubios HRV, Version 2.0. Biosignal Analysis and Medical Imaging Group (BSAMIG). Department of Physics, University of Kuopio, Kuopio. http://kubios.uef.fi

[41] Van Roon, A.M. (1998) Short-Term Cardiovascular Effects of Mental Tasks. PhD Thesis, University of Groningen, Groningen.

[42] Cohen, J. (1988) Statistical Power Analysis for the Behavioral Sciences. 2nd Edition, Lawrence Erlbaum Associates, Hillsdale.

[43] Harmat, L., de Manzano, O., Theorell, T., Högman, L., Fischer, H. and Ullén, F. (2015) Physiological Correlates of the Flow Experience during Computer Game Playing. International Journal of Psychophysiology, 97, 1-7. http://dx.doi.org/10.1016/j.ijpsycho.2015.05.001

[44] Hansen, A.L., Johnsen, B.H. and Thayer, J.F. (2003) Vagal Influence on Working Memory and Attention. International Journal of Psychophysiology, 48, 263-274. http://dx.doi.org/10.1016/S0167-8760(03)00073-4

[45] Csíkszentmihályi, M. (1996) Creativity: Flow and the Psychology of Discovery and Invention. Harper Collins, New York, 111-113. 